\title{
Amblyomma aureolatum (Acari: Ixodidae): nuevos registros en las provincias de Buenos Aires y Santa Fe, Argentina
}

\author{
Colombo VC ${ }^{1 *}$, Pereira J ${ }^{2}$, Nava $\mathrm{S}^{3}$, Beldomenico PM ${ }^{1}$ \\ 1 Laboratorio de Ecología de Enfermedades, Instituto de Ciencias Veterinaria del Litoral, UNL-CONICET \\ 2 CONICET - Museo Argentino de Ciencias Naturales "Bernardino Rivadavia". División Mastozoología. Buenos Aires, \\ Argentina \\ 3 Instituto Nacional de Tecnología Agropecuaria, Estación Experimental Agropecuaria Rafaela, Provincia de Santa Fe, \\ Argentina
}

* Correspondencia: VC Colombo. Laboratorio de Ecología de Enfermedades. Instituto de Ciencias Veterinarias del Litoral (UNL-CONICET). Facultad de Ciencias Veterinarias, RP Kreder 2805, 3080 Esperanza, Santa Fe, Argentina. E-mail: valecc1983@yahoo.com.ar

Recibido: 16 Junio 2016. Aceptado: 24 Julio 2016. Disponible en línea: 26 Julio 2016

Editor: J. M. Venzal

\begin{abstract}
RESUMEN. En los meses de julio de 2015 y enero de 2016 se colectaron garrapatas de un gato montés (Leopardus geoffroyi) localizado en la provincia de Buenos Aires y de un canino doméstico (Canis lupus familiaris) en la provincia de Santa Fe, respectivamente. El objetivo del presente trabajo es reportar el primer registro de Amblyomma aureolatum en la provincia de Buenos Aires y el primer hallazgo sobre caninos domésticos de la provincia de Santa Fe. Amblyomma aureolatum presenta importancia sanitaria ya que ha sido implicada como vector de Rickettsia rickettsii, uno de los agentes causales del grupo de las fiebres manchadas en humanos, y de Rangelia vitalii, agente causal de piroplasmosis en caninos domésticos, enfermedad vulgarmente conocida como "nambiuvú". El presente reporte es un alerta para la comunidad sobre la presencia de $A$. aureolatum en nuevas localidades con el fin de implementar medidas profilácticas en caninos domésticos, para prevenir la infestación por este parásito y así evitar posibles patologías asociadas.
\end{abstract}

SUMMARY. Amblyomma aureolatum (Acari: Ixodidae): new records in Buenos Aires and Santa Fe province, Argentina. In July of 2015 and January of 2016, ticks were recovered from a Geoffroy's cat (Leopardus geoffroyi) in Buenos Aires province and from a domestic dog (Canis lupus familiaris) in Santa Fe province, respectively. All ticks were identified as Amblyomma aureolatum. This report is the first record of A. aureolatum in Buenos Aires province and the first record in domestic dogs of Santa Fe province. Amblyomma aureolatum is a recognized vector of Rickettsia rickettsii, a spotted fever group agent in humans, and of Rangelia vitalii, a pathogenic piroplasmid of dogs, commonly referred to as "nambiuvú". These findings allow local people to be aware of the presence of $A$. aureolatum to prevent infestations in dogs, and thus avoiding the occurrence of tick-borne diseases.

Palabras clave: Amblyomma aureolatum; perro doméstico; Leopardus geoffroyi; Santa Fe; Buenos Aires; Argentina

Key words: Amblyomma aureolatum; domestic dog; Leopardus geoffroyi; Santa Fe; Buenos Aires; Argentina

Amblyomma aureolatum Pallas, 1772 (Acari: Ixodidae) se distribuye en la región Neotropical, principalmente en el área este de Sudamérica incluyendo los países de Argentina, Brasil, Guyana Francesa, Paraguay, Surinam y Uruguay (Figura 1) (Guglielmone et al., 2003). En Argentina existen registros en las provincias de Misiones, Chaco, Entre Ríos, Santa Fe y Ciudad Autónoma de Buenos Aires (Figura y Tabla 1) (Boero, 1954, 1957; Ivancovich, 1973; Guglielmone et al., 2002; Tarragona et al., 2012; Cicuttin et al., 2013; Gervasoni et al., 2013). Los hospedadores principales para los adultos de $A$. aureolatum son carnívoros silvestres y domésticos de las familias Canidae y Felidae, así como prociónidos silvestres, mientras que los estadios inmaduros utilizan como hospedadores más usuales a las aves paseriformes (Guglielmone et al., 2003; Arzua et al. 2003, 2005; Venzal et al., 2005; Ogrzewalska et al. 2012). Aunque no sea su hospedador principal, existen registros del parasitismo de $A$. aureolatum en seres humanos (Guglielmone et al., 2006; Saravia et al., 2014).

Amblyomma aureolatum presenta importancia sanitaria ya que ha sido implicada como vector de Rickettsia rickettsii, uno de los agentes causales del grupo de las fiebres manchadas en humanos (Labruna et al., 2011), y de Rangelia vitalii, agente causal de piroplasmosis en caninos domésticos, enfermedad vulgarmente conocida como "nambiuvú" (Soares, 2014). En la Argentina, casos de fiebre manchada en humanos asociados a la transmisión de $R$. rickettsii por Amblyomma cajennense sensu lato han sido detectados en el noroeste del 
país (Paddock et al., 2008), y caninos domésticos infectados por $R$. vitalii fueron detectados en las provincias de Misiones y Entre Ríos (Eiras et al., 2014; Sánchez et al., 2015).

A pesar de que A. aureolatum fue previamente registrada en carnívoros silvestres (Procyon cancrivorus y Puma yagouaroundi) de la provincia de Santa Fe (Tarragona et al., 2012; Gervasoni et al., 2013), hasta la fecha no existen registros de la asociación de $A$. aureolatum con animales domésticos en esta provincia. En la provincia de Buenos Aires no se ha registrado previamente la presencia de esta garrapata, en cambio sí en caninos domésticos de la Ciudad Autónoma de Buenos Aires (Cicuttin et al., 2013) (La Ciudad Autónoma de Buenos Aires no pertenece a la provincia del mismo nombre). El objetivo del presente trabajo es reportar el primer registro de A. aureolatum en la provincia de Buenos Aires y su primer hallazgo en caninos domésticos de la provincia de Santa Fe.

En julio de 2015 se colectaron garrapatas de un ejemplar de gato montés (Leopardus geoffroyi) capturado en el marco de un proyecto de ecología de la especie desarrollado en la Estación Experimental Agropecuaria Delta (EEA) del Instituto Nacional de Tecnología Agropecuaria (INTA) $\left(34^{\circ} 08^{\prime} S, 58^{\circ} 53^{\prime} \mathrm{O}\right)$, en el partido de Campana, provincia de Buenos Aires, y durante enero de 2016 a partir de un canino doméstico (Canis lupus familiaris) localizado en la costa del Paraje Chaco Chico, distrito de Monte Vera, departamento de La Capital, provincia de Santa Fe (31ㅇ 33'S, 60 으으. Argentina.

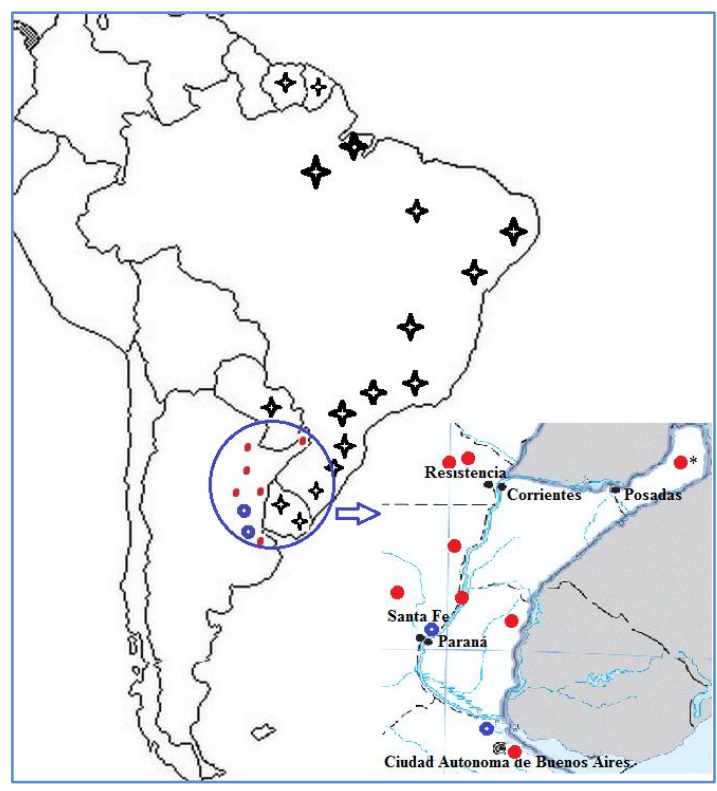

Figura 1. Áreas en donde especímenes de $A$. aureolatum fueron colectados en Sudamérica $(\downarrow)$ y Argentina.

( Registros previos, $\bullet$ Registros reportados en el presente trabajo). * El registro para la provincia de Misiones carece de datos de localidad y hospedador.
Tabla 1: Localidades, hospedadores y referencias bibliográficas correspondientes a los registros previos de $A$. aureolatum en Argentina.

\begin{tabular}{|c|c|c|}
\hline Localidad & Hospedadores & Referencia \\
\hline $\begin{array}{l}\text { La Paz, Entre Ríos } \\
\left(30 \circ 45^{\prime} \text { S } 59 \text { 은 }\right. \\
\left.39^{\prime} \mathrm{O}\right)\end{array}$ & Cerdocyon thous & $\begin{array}{l}\text { Boero, } 1954 \text { ( } A \text {. } \\
\text { aureolatum es } \\
\text { nombrada como } A \text {. } \\
\text { striatum) }\end{array}$ \\
\hline $\begin{array}{l}\text { Misiones } \\
\text { (localidad } \\
\text { desconocida) }\end{array}$ & Desconocido & $\begin{array}{l}\text { Boero, } 1957 \text { ( } A \text {. } \\
\text { aureolatum es } \\
\text { nombrada como } A \text {. } \\
\text { striatum) }\end{array}$ \\
\hline 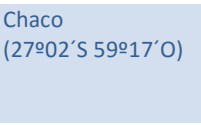 & Leopardus geoffroyi & $\begin{array}{l}\text { Ivancovich, } 1973 \text { ( } A \text {. } \\
\text { aureolatum es } \\
\text { nombrada como } A \text {. } \\
\text { striatum) }\end{array}$ \\
\hline 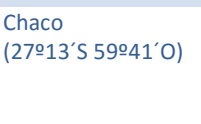 & Puma concolor & $\begin{array}{l}\text { Ivancovich y Luciani, } \\
1992 \text { (A. aureolatum es } \\
\text { nombrada como } A \text {. } \\
\text { striatum) }\end{array}$ \\
\hline 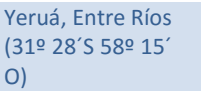 & Canino doméstico & $\begin{array}{l}\text { Guglielmone et al., } \\
2002\end{array}$ \\
\hline $\begin{array}{l}\text { Reconquista, } \\
\text { Santa Fe } \\
\left(29^{\circ} 38^{\prime} \mathrm{S}, 59^{\circ} 46^{\prime}\right. \\
\text { O) }\end{array}$ & Procyon cancrivorus & Tarragona et al., 2012 \\
\hline $\begin{array}{l}\text { Ciudad Autónoma } \\
\text { de Buenos Aires } \\
\left(34^{\circ} 36^{\prime} \text { S } 58^{\circ} 20^{\prime}\right. \\
\text { O) }\end{array}$ & $\begin{array}{l}\text { Canino doméstico } \\
\text { Poospiza nigrorufa }\end{array}$ & Cicuttin et al., 2013 \\
\hline $\begin{array}{l}\text { San Cristobal, } \\
\text { Santa Fe } \\
\left(30^{\circ} 19^{\prime} \mathrm{S} 61^{\circ} 14^{\prime} \mathrm{O}\right)\end{array}$ & Puma yagouaroundi & Gervasoni et al., 2013 \\
\hline
\end{tabular}

Ambos sitios forman parte de la eco-región Delta e Islas del Paraná (Burkart et al., 1999). La EEA Delta del INTA se encuentra sobre el margen del rio Paraná a 75 $\mathrm{km}$ de la Ciudad de Buenos Aires, en un área formada por islas grandes fragmentadas por canales de navegación artificiales, con presencia de albardones que rodean bajos inundables. La vegetación dominante está formada por gramíneas, bosques de Erythrina crista-galli y áreas de pajonales (Kandus, 1997). La actividad forestal es la más desarrollada con plantaciones de Populus spp. y Salix spp., aunque también se desarrolla la actividad ganadera de cría.

La costa del paraje Chaco Chico se caracteriza por ser una zona de casas de fin de semana en los márgenes de la laguna Setúbal, con una comunidad residente de menos de 50 habitantes, ubicada a $12 \mathrm{~km}$ al norte de la ciudad de Santa Fe. Sobre albardones arcillosos se desarrolla una vegetación conformada principalmente por leñosas como Sapium haematospermum, Salix humboldtiana, Peltophorum dubium, Erythrina cristagalli, Acacia caven, Parkinsonia aculeata, entre otros, intercalados con arbustos, enredaderas y herbáceas (Pautasso, 2008). Durante los fines de semana el paraje es visitado por decenas de personas que utilizan las playas de la laguna a modo recreativo. La fauna de ambos sitios está conformada por diversas especies de aves, anfibios, marsupiales, coipos, pequeños roedores, carnívoros silvestres y animales domésticos; contando además en el área de la EEA Delta del INTA con Hydrochoerus hydrochaeris y Blastocerus 
dichotomus (Canevari y Vaccaro, 2007; Pautasso, 2008).

Las garrapatas colectadas fueron conservadas en etanol 960 y se determinaron siguiendo la clave de Guglielmone y Viñabal (1994) ( $A$. aureolatum es nombrada como $A$. striatum) y mediante la comparación con material de referencia depositado en la Colección de Garrapatas del Instituto Nacional de Tecnología Agropecuaria, Estación Experimental Agropecuaria Rafaela. Todos los individuos fueron ingresados en la colección de Ixodidos de la Facultad de Ciencias Veterinarias, Universidad Nacional del Litoral, Argentina (códigos de acceso: SF00108b, SF00108c, BA00006).

Las garrapatas colectadas fueron identificadas como 3 especímenes adultos de la especie $A$. aureolatum: sobre el canino doméstico se colectaron 1 hembra y 1 macho y sobre L. geoffroyi, 1 hembra. La determinación de los especímenes colectados se basó en la combinación de los siguientes caracteres morfológicos: contorno corporal oval alargado, surco marginal incompleto cubriendo solo dos festones (en machos), escudo de color castaño claro con puntuaciones profundas, numerosas y uniformemente distribuidas, hipostoma espatulado con dentición $3 / 3$, y coxa I con dos espinas sub-iguales y extremadamente largas que alcanzan la parte media de la coxa II con la espina externa terminando en una punta recta. Para determinar si las garrapatas pertenecían a la especie $A$. ovale o A. aureolatum (especies morfológicamente similares) se utilizaron los caracteres morfológicos diferenciales descriptos por Aragão y Fonseca (1961), con énfasis en las diferencias en la coloración del escudo y las características de la espina externa de la coxa II.

El presente hallazgo representa el primer registro de A. aureolatum parasitando caninos domésticos en la provincia de Santa $\mathrm{Fe}$, y el primer hallazgo de esta garrapata en la provincia de Buenos Aires, en este caso, asociada a $L$. geoffroyi. Como se mencionó en la introducción, tanto en el Chaco Chico como en el INTA EEA Delta, existe actividad humana frecuente en convivencia con los animales silvestres del lugar, existiendo diversas oportunidades de interacción entre animales silvestres, domésticos y seres humanos, aumentando así las posibilidades de parasitismo de $A$. aureolatum en seres humanos $y$, por ende, de la transmisión de posibles patógenos. Así, el presente reporte permite alertar sobre la presencia de este parásito con el fin de implementar medidas preventivas tanto en la comunidad, para evitar el contacto directo con $A$. aureolatum, como en caninos domésticos para prevenir su infestación, evitando posibles patologías asociadas.

Se ha postulado que las aves posiblemente actúen como dispersores geográficos de los estadios inmaduros de A. aureolatum (Ogrzewalska et al., 2016), cuyos estadios adultos entrarían luego en contacto con mamíferos silvestres y domésticos. Así los caninos domésticos podrían facilitar el contacto de $A$. aureolatum, y de los patógenos que potencialmente transmiten, con los seres humanos. Por otro lado, $A$. aureolatum actúa como vector de $R$. vitalii produciendo en caninos domésticos un cuadro febril caracterizado por ictericia, anemia y hemorragias. Estudios en Brasil permitieron postular que caninos silvestres podrían actuar como reservorios de $R$. vitalii, ya que se detectó la presencia del patógeno en animales asintomáticos, amplificando el número de $A$. aureolatum infectadas que potencialmente pueden parasitar a caninos do-mésticos (Soares, 2014). En la actualidad, en la Argentina se desconoce la existencia de reservorios de la enfermedad y como acontece la diseminación a animales domésticos.

Por último, es posible que la distribución de $A$. aureolatum en Argentina sea mayor a la actualmente conocida, pero probablemente restringida a regiones cuyas características de humedad y altitud sean compatibles con los requerimientos de esta especie (Guglielmone y Nava, 2014, Barbieri et al., 2015). Los reportes de $A$. aureolatum en Santa Fe y Ciudad Autónoma de Buenos aires corresponden a un escaso número de hallazgos ocasionales (Tarragona et al., 2012; Cicuttin et al., 2013; Gervasoni et al., 2013), por lo que futuros estudios que incluyan un muestreo de un mayor número de hospedadores y de áreas, así como la detección de Rickettsia spp. y $R$. vitalii en los especímenes colectados, permitirán conocer con mayor precisión la situación actual de A. aureolatum y de los patógenos que transmiten.

\section{Bibliografía}

Aragão H \& Fonseca FD. 1961. Notas de ixodologia: IX. O complexo ovale do gênero Amblyomma. Mem. Inst. Oswaldo Cruz, 59: 131-148.

Arzua M, Navarro da Silva M, Famadas KM, Beati L, Barros Battesti DM. 2003. Amblyomma aureolatum and Ixodes auritulus (Acari: Ixodidae) on birds in southern Brazil, with notes on their ecology. Exp. Appl. Acarol. 31: 283-6.

Arzua M, Onofrio VC, Barros-Battesti DM. 2005. Catalogue of the tick collection (Acari, Ixodida) of the Museu de História Natural Capao da Imbuia, Curitiba, Paraná, Brazil. Rev. Bras. Zool. 22: 623-32.

Barbieri JM, Da Rocha CM, Bruhn FRP, Cardoso DL, Pinter A \& Labruna MB. 2015. Altitudinal assessment of Amblyomma aureolatum and Amblyomma ovale (Acari: Ixodidae), vectors of spotted fever group rickettsiosis in the state of São Paulo, Brazil. J. Med. Entomol. 52: 1170-1174.

Boero JJ. 1954. Los ixodideos de la República Argentina y sus huéspedes. Rev. Fac. Agr. Vet. 13: 505-514. 
Boero JJ. 1957. Las garrapatas de la República Argentina (Acarina: Ixodoidea). Dpto. Edit. Univ. Buenos Aires. 113 pp.

Burkart R, Bárbaro N, Sánchez RO \& Gómez DA. 1999. Ecoregiones de la Argentina. APN, Prodia, Buenos Aires, 43 pp.

Canevari M \& Vaccaro O. 2007. Guía de mamíferos del sur de América del Sur. Ed. LOLA, Buenos Aires. 424 pp.

Cicuttin GL, Sassaroli JC, Ardiles MI, Zotter AC, Guglielmone AA \& Nava S. 2013. Presencia de dos especies de garrapatas (Acari: Ixodidae) con importancia médica en la Ciudad de Buenos Aires. Medicina (Buenos Aires). 73: 389-390.

Eiras DF, Craviotto MB, Baneth G \& Moré G. 2014. First report of Rangelia vitalii infection (canine rangeliosis) in Argentina. Parasitol. Int. 63:729-734.

Gervasoni SH, Ruiz MF \& Sciabarrasi AA. 2013. Nuevo registro de Amblyomma aureolatum (Pallas, 1772) (Acari: Ixodidae), en la provincia de Santa Fe, Argentina. XIV Jornadas de Divulgación Técnico-Científicas 2013. Facultad de Ciencias Veterinarias - Universidad Nacional de Rosario.

Guglielmone AA \& Viñabal AE. 1994. Claves morfológicas dicotómicas e información ecológica para la identificación de garrapatas del género Amblyomma Koch, 1844 de la Argentina. Rev. Inv. Agropec. 25: 3967.

Guglielmone AA, Mangold AJ, Boero C \& Azcure R. 2002. Nuevo registro de Amblyomma aureolatum (Pallas, 1772) (= Amblyomma striatum Koch, 1844) en Entre Ríos, Argentina. Rev. FAVE-Ciencias Veterinarias. 1: 3335.

Guglielmone AA, Estrada Peña A, Mangold AJ, Barros Battesti DM, Labruna MB, Martins JR, Venzal JM, Arzua M \& Keirans JE. 2003. Amblyomma aureolatum (Pallas, 1772) and Amblyomma ovale Koch, 1844 (Acari: (xodidae): host, distribution and 16S rDNA sequences. Vet. Parasitol.113: 273-288.

Guglielmone AA, Beati L, Barros-Battesti DM, Labruna MB, Nava S, Venzal JM, Mangold AJ, Szabó PJ, Martins JR, González-Acuña D \& Estrada-Peña A. 2006. Ticks (Ixodidae) on humans in South America. Exp. App. Acarol. 40: 83-100

Guglielmone AA \& Nava S. 2014. Distribución geográfica, hospederos y variabilidad genética de Amblyomma ovale y Amblyomma aureolatum [Acari: Ixodidae], dos vectores potenciales de rickettsias en la Argentina. (pp. 313 - 319). En: Farjat JB, Enría D, Martino P, Rosenzvit M, Seijo A. (eds.). Temas de zoonosis VI. Ed. Asociación Argentina de Zoonosis, Buenos Aires.

Ivancovich JC. 1973. Las garrapatas del Chaco y Formosa. Primera comunicación. Rev. Inv. Agropec. Ser. 4, 10: 924.

Ivancovich JC \& Luciani CA. 1992. Las garrapatas de Argentina. Monogr. Asoc. Arg. Parasitol. Vet. 95

Kandus P. 1997. Análisis de Patrones de vegetación a escala regional en el Bajo Delta del Río Paraná (Argentina). Tesis Doctoral. Universidad de Buenos Aires, Argentina. 235 pp.
Labruna MB, Ogrzewalska M, Soares JF, Martins TF, Soares HS, Moraes-Filho J, Nieri-Bastos FA, Almeida AP \& Pinter A. 2011. Experimental infection of Amblyomma aureolatum ticks with Rickettsia rickettsii. Emerg. Infect. Dis.17: 829-34.

Ogrzewalska M, Saraiva DG, Moraes-Filho J, Martins TF, Costa FB, Pinter A \& Labruna M. 2012. Epidemiology of Brazilian spotted fever in the Atlantic Forest, state of São Paulo, Brazil. Parasitology. 139: 1283-300.

Ogrzewalska M, Schwarcz K, Bajay MM, Bajay SK, Pinheiro JB, Zucchi MI, Pinter A \& Labruna, M. B. 2016. Characterization of Genetic Variability and Population Structure of the Tick Amblyomma aureolatum (Acari: Ixodidae). J. Med. Entomol. doi: 10.1093/jme/tjw049

Paddock CD, Fernandez S, Echenique GA, Sumner JW, Reeves WK, Zaki SR \& Remondegui CE. 2008. Rocky mountain spotted fever in Argentina. Am. J. Trop. Med. Hyg. 78: 687-692.

Pautasso AA. 2008. Mamíferos de la provincia de Santa Fe, Argentina. Ed. Comunicaciones del Museo Provincial de Ciencias Naturales "Florentino Ameghino", Santa Fe. 248 pp.

Sánchez RO, Moré GA, Eiras DF. 2015. Piroplasmosis canina por Rangelia vitalii (Protozoa, Piroplasmida) en la ciudad de Concordia, Entre Ríos. Su informe Entre Rios. 139: 27-30.

Saraiva DG, Soares HS, Soares JF \& Labruna MB. 2014. Feeding period required by Amblyomma aureolatum ticks for transmission of Rickettsia rickettsii to vertebrate hosts. Emerg. Infect. Dis. 20: 1504-1510.

Soares JF. 2014. História natural da rangeliose. Tesis Doctoral. Faculdade de Medicina Veterinárias y Zootecnia. Universidade de São Paulo, Brasil. 121 pp.

Tarragona EL, Eberhardt MAT, Zurvera D, Beldomenico PM \& Mastropaolo M. 2012. Primer registro de Amblyomma aureolatum (Pallas, 1772) y Amblyomma Ovale Koch, 1844 (Acari: Ixodidae) en la provincia de Santa Fe, Argentina. FAVE Sección Ciencias Veterinarias. 11: 5963.

Venzal JM, Félix ML, Olmos A, Mangold AJ, Guglielmone AA. 2005. A collection of ticks (Ixodidae) from wild birds in Uruguay. Exp. Appl. Acarol. 36: 325-331. 